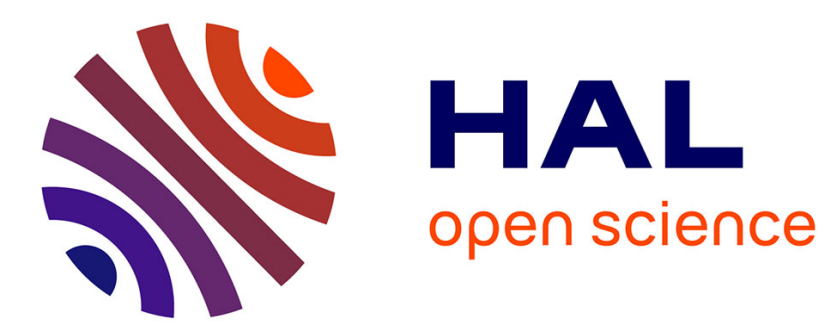

\title{
High-field magnetoresistance and quantum effects in the organic compound (TMTSF)2PF6
}

J.P. Ulmet, P. Auban, A. Khmou, S. Askenazy, A. Moradpour

\section{To cite this version:}

J.P. Ulmet, P. Auban, A. Khmou, S. Askenazy, A. Moradpour. High-field magnetoresistance and quantum effects in the organic compound (TMTSF)2PF6. Journal de Physique Lettres, 1985, 46 (12), pp.535-542. 10.1051/jphyslet:019850046012053500 . jpa-00232556

\section{HAL Id: jpa-00232556 https://hal.science/jpa-00232556}

Submitted on 1 Jan 1985

HAL is a multi-disciplinary open access archive for the deposit and dissemination of scientific research documents, whether they are published or not. The documents may come from teaching and research institutions in France or abroad, or from public or private research centers.
L'archive ouverte pluridisciplinaire HAL, est destinée au dépôt et à la diffusion de documents scientifiques de niveau recherche, publiés ou non, émanant des établissements d'enseignement et de recherche français ou étrangers, des laboratoires publics ou privés. 
Classification

Physics Abstracts

$72.80 \mathrm{~L}-72.20 \mathrm{M}-71.25 \mathrm{H}$

\title{
High-field magnetoresistance and quantum effects in the organic compound (TMTSF) ${ }_{2} \mathbf{P F}_{6}$
}

\author{
J. P. Ulmet, P. Auban, A. Khmou, S. Askenazy \\ Laboratoire de Physique des Solides (*) et Service des Champs Magnétiques Intenses, INSA, \\ Avenue de Rangueil, 31077 Toulouse Cedex, France
}

and A. Moradpour

Laboratoire de Physique des Solides, Université Paris-Sud, 91405 Orsay Cedex, France

(Reçu le 28 décembre 1984, accepté sous forme définitive le 29 avril 1985)

\begin{abstract}
Résumé. - Nous avons étudié la magnétorésistance transverse du (TMTSF) ${ }_{2} \mathrm{PF}_{6}$ pour des champs magnétiques allant jusqu'à $32 \mathrm{~T}$, à basse température jusqu'à $4,2 \mathrm{~K}$. Nous observons des oscillations de Shubnikov-de-Haas directement sur la magnétorésistance pour des champs supérieurs à $18 \mathrm{~T}$, mais il n'est pas du tout certain qu'il existe un champ seuil car la disparition des oscillations peut être expliquée par l'amortissement normal fonction du champ magnétique. Par contre, celles-ci s'évanouissent très rapidement entre 5 et $4,2 \mathrm{~K}$. Ce comportement inhabituel pourrait être dû à une transition de phase. Enfin nous donnons une exploration du champ fondamental en fonction de $\theta\left(\theta=\right.$ angle entre $B$ et l'axe $\left.c^{*}\right)$ qui montre le gondolement des «tubes de Fermi » le long de l'axe $c^{*}$.
\end{abstract}

\begin{abstract}
The transverse magnetoresistance of (TMTSF) ${ }_{2} \mathrm{PF}_{6}$ has been investigated in magnetic fields up to $32 \mathrm{~T}$, at low temperatures down to $4.2 \mathrm{~K}$. Shubnikov-de-Haas oscillations are directly observed on the magnetoresistance in fields higher than $18 \mathrm{~T}$, but there is no evidence of a threshold field since the disappearance of oscillations can be explained by the normal field-dependent damping. However these oscillations vanish very quickly between 5 and $4.2 \mathrm{~K}$. This unusual behaviour could be related to a phase-transition. Finally an exploration of the fundamental field versus $\theta$ is given $\left(\theta=\right.$ angle between $B$ and $\left.c^{*}\right)$, showing the warping of the "Fermi tubes" along the $c^{*}$ axis.
\end{abstract}

\section{Introduction.}

The organic compound (TMTSF) ${ }_{2} \mathrm{PF}_{6}$ was the first organic conductor to exhibit superconductivity under pressure [1]. Its resistivity has a metallic behaviour down to $\simeq 17 \mathrm{~K}$. After a minimum around this temperature, it increases at low temperatures (semi-insulating state). In this region the compound is believed to be in a spin-density-wave (SDW) phase [2, 3]. Oscillations of the magnetoresistance have been seen by Kwak et al., in rather low fields and under pressure [4]. These oscillations were first attributed to the Shubnikov-de-Haas (SdH) effect, but it seems now, in the light of recent developments, that they could be related to different phase transitions inside the SDW region, as in (TMTSF) ${ }_{2} \mathrm{ClO}_{4}[5,6]$. We report the first observation of $\mathrm{SdH}$ effect in very high fields $(B>18 \mathrm{~T})$ at zero pressure, for various angles between $B$ (magnetic field) and the

(*) Laboratoire associé au CNRS. 
$c^{*}$ axis, allowing a direct spectroscopy of the Fermi surface (FS). The sudden disappearance of oscillations when $T$ is lowered under $5 \mathrm{~K}$ is correlated to the possibility of a phase transition

around this temperature. Concerning magnetoresistance we should say that the behaviour of (TMTSF) ${ }_{2} \mathrm{PF}_{6}$ is rather similar to that of (TMTSF) $)_{2} \mathrm{ClO}_{4}$ within the same experimental range [7, 8].

\section{Experimental details.}

The sample is mounted on four gold wires $(17.5 \mu \mathrm{m}$ of diameter $)$ with gold paint. The sampleholder is mounted on a rotating device which allows at least a $360^{\circ}$ rotation of the angle between the field $B$ and the crystalline axis. Angles are set within an accuracy of about $\pm 2^{\circ}$. A slowly decreasing pulsed field (crow-bar type) reaching $35 \mathrm{~T}$ is used [9].

The sample is fed with a constant amplitude alternating current, low enough to avoid heating or non-ohmic effects flowing along the $a$ axis. After amplification, filtering and demodulation, the signal is driven to a storage oscilloscope in parallel with a computer.

\section{Results and discussion.}

The sample is slowly cooled (about $1 \mathrm{~K} / \mathrm{min}$ ) from room-temperature down to $4.2 \mathrm{~K}$. The lowtemperature resistivity, free from jumps, is given in figure 1 , showing the " metal-insulator transition » around $17 \mathrm{~K}$.

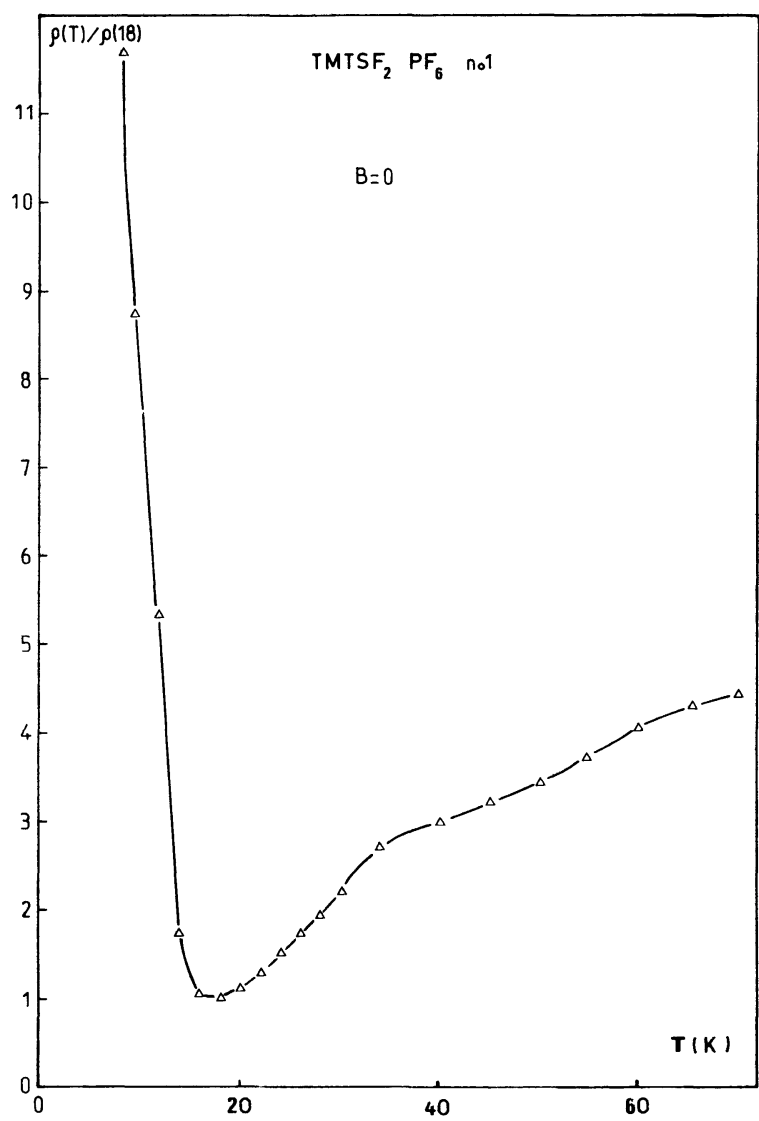

Fig. 1. - Zero field resistivity along the $a$-direction from $80 \mathrm{~K}$ down to $10 \mathrm{~K}$ normalized to its minimum value (for $T=18 \mathrm{~K}$ ). 
3.1 ANISOTROPY OF THE TRANSVERSE MAGNETORESISTANCE. - The transverse magnetoresistance taken at $B=5 \mathrm{~T}$ is plotted against the angle $\theta=\left(B, b^{\prime}\right)$, the latter varying by steps of $10^{\circ}$. Figure 2 gives three of these curves corresponding to the temperatures $T=4.2 ; 8$; $16 \mathrm{~K}$. As in (TMTSF) ${ }_{2} \mathrm{ClO}_{4}$ the minimum is assigned to the $b^{\prime}$ direction (projection of $b$ on a plane perpendicular to $a)$ [4] and is noted $\theta=0$. The anisotropy ratio $r=\left(\Delta \rho / \rho_{0}\right)_{\max } /\left(\Delta \rho / \rho_{0}\right)_{\min }$ decreases with increasing temperature. The angle between the magnetoresistance extrema is close to $85^{\circ}$ (or $95^{\circ}$ ).

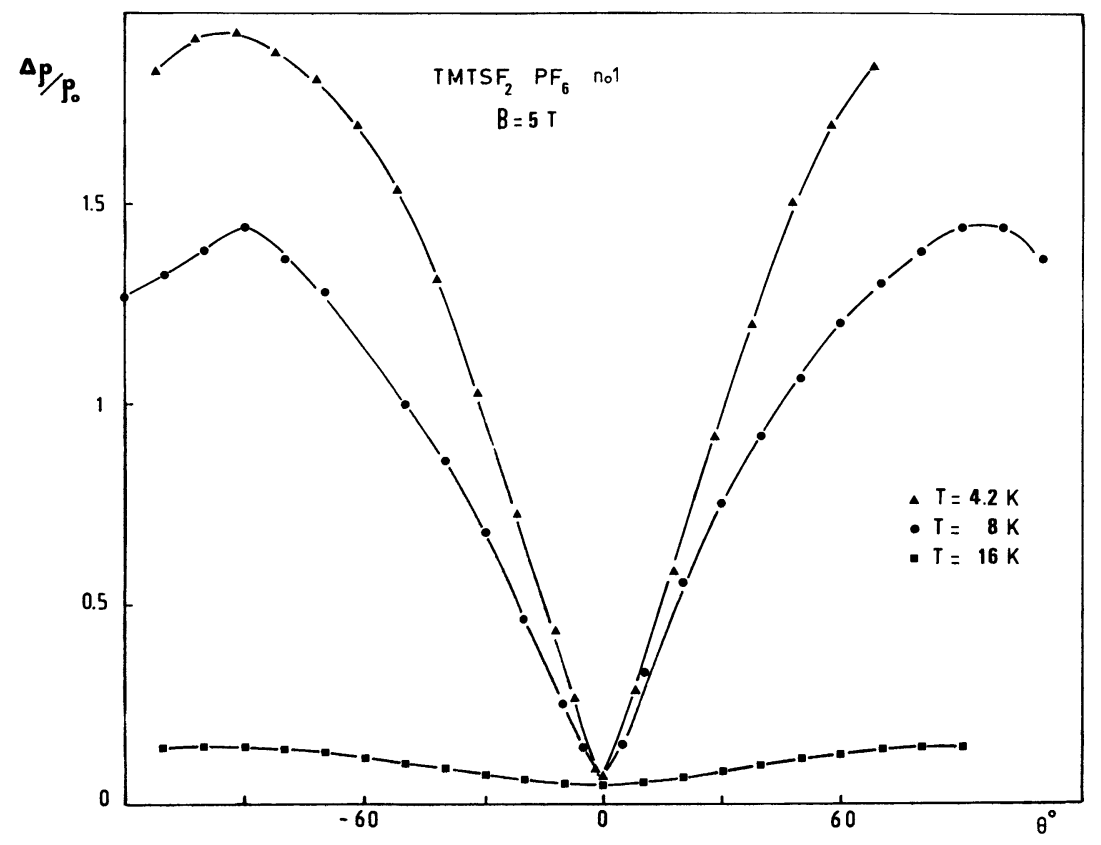

Fig. 2. - Transverse magnetoresistance anisotropy for $B=5 \mathrm{~T}$ and $T=4.2,8,16 \mathrm{~K}$.

3.2 ShubNiKov-DE-HAAS EFFeCT. - If the field is along the $b^{\prime}$ direction no closed orbit can be found, indicating an open FS along the $c^{*}$ direction. A magnetoresistance with a tendency to saturation is actually observed. On the contrary, when $B$ is applied along $c^{*}$, closed orbits are possible on the FS, due to nesting. Thus the Fermi surface can be described as a system of warped tubes lying along the $c^{*}$ direction. Magnetoresistance then increases with $B$ as we have hole and electron pockets [10]. In its high field part SdH oscillations are directly visible (Fig. 3).

The area $S$ of the FS cross-section perpendicular to $B$ is proportional to the fundamental field $B_{\mathrm{F}}$.

$$
\frac{1}{B_{\mathrm{F}}}=\frac{2 \pi e}{\hbar S}
$$

If we call $B_{N}$ the field positions of the SdH oscillations, we can plot $\frac{1}{B_{N}}$ against the quantum number $N$. As we have the linear relation :

$$
\frac{1}{B_{N}}=\frac{1}{B_{\mathrm{F}}}\left(N-N_{0}\right),
$$




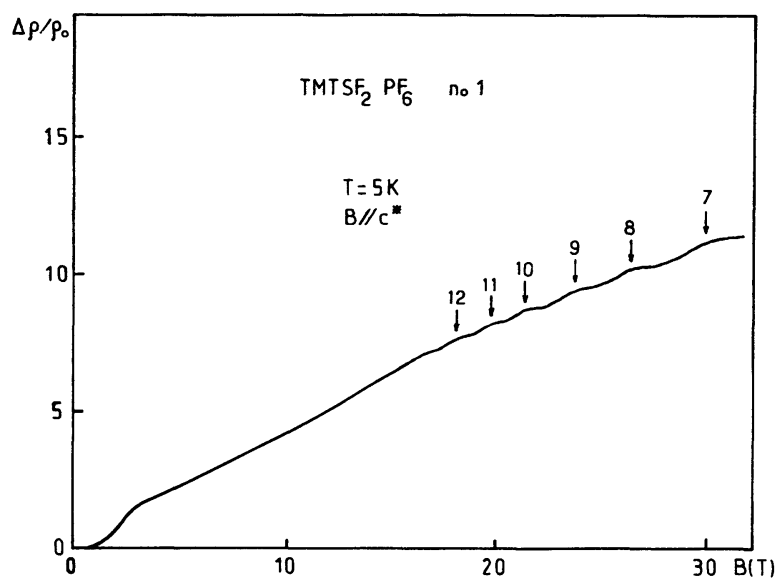

Fig. 3. - Transverse magnetoresistance for $T=5 \mathrm{~K}$ and $\theta=-90^{\circ}\left(B / / c^{*}\right)$.

we can use a mean square method to determine the best straight line corresponding to the experimental points. The fundamental field $B_{\mathrm{F}}$ of the oscillations is simply given by the inverse of the slope. In figure 4 we give an example of such a determination for $\theta=-90^{\circ}\left(B / / c^{*}\right)$. For $B_{\mathrm{F}}=$ $230 \mathrm{~T}$ at $\theta=-90^{\circ}$, the area $S$ is close to $3 \%$ of the Brillouin zone area (in the $a b$ plane). These values are to be compared with those of (TMTSF) ${ }_{2} \mathrm{ClO}_{4}$ where a slightly higher fundamental field is observed, leading to a cross-section of $3.4 \%$ of the Brillouin zone area [7]. Different wavevectors are theoretically possible to nest the FS. However from our data, using the calculations of Kwak [11], the nesting vector could be $q=\left(2 k_{\mathrm{F}}, 0\right)$, implying equal pockets of electrons and holes. The values of $B_{\mathrm{F}}$ for different angles $\theta$ are directly related to the angular variations of $S$.

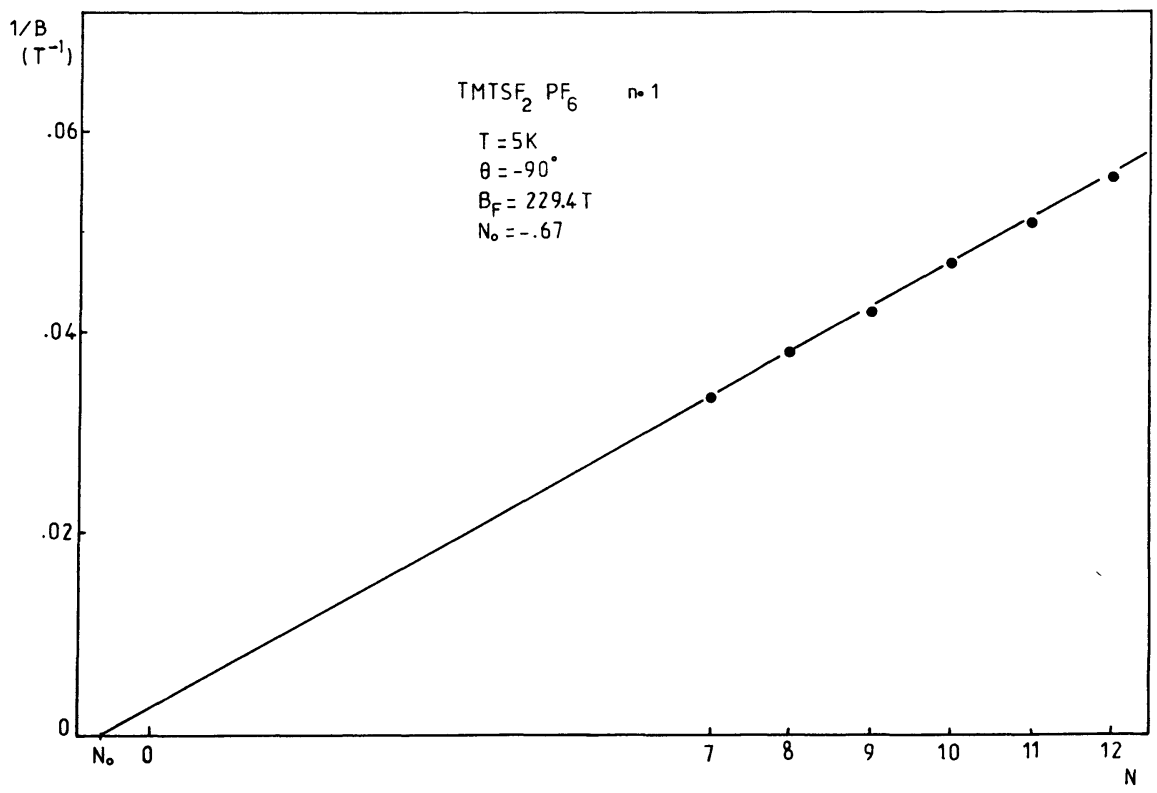

Fig. 4. - Inverses of oscillation fields $B_{N}$ plotted against the quantum numbers $N$ for $T=5 \mathrm{~K}$ and $\theta=-90^{\circ}$ $\left(B / / c^{*}\right)$, giving the fundamental field $B_{\mathrm{F}}$ and the phase $N_{0}$. 
We have studied two different samples of (TMTSF) ${ }_{2} \mathrm{PF}_{6}$. The curves $B_{\mathrm{F}}(\theta)$ given in figures 5 and 6 respectively look very similar. Some striking points can be noticed from these results :

a) For $B / / c^{*}$ the fundamental fields are almost the same for both samples, $B_{\mathrm{F}} \simeq 229 \mathrm{~T}$. Moving away from $c^{*}$, the $B_{\mathrm{F}}$ determination becomes less and less accurate as the number of visible oscillations decreases as well as their amplitude. However the corresponding values of $B_{\mathrm{F}}$ remain rather close for the two samples within the experimental precision.

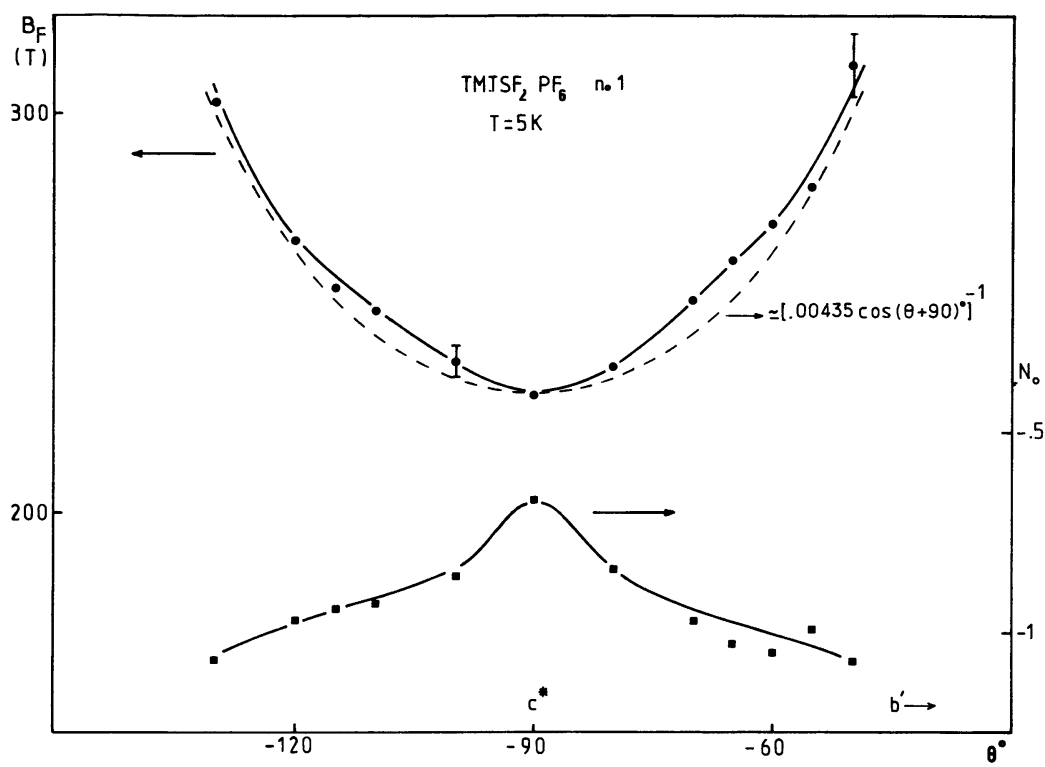

Fig. 5. - Angular dependence of the fundamental field $B_{\mathrm{F}}$ and of the phase $N_{0}$ for $T=5 \mathrm{~K}$, on sample no 1 .

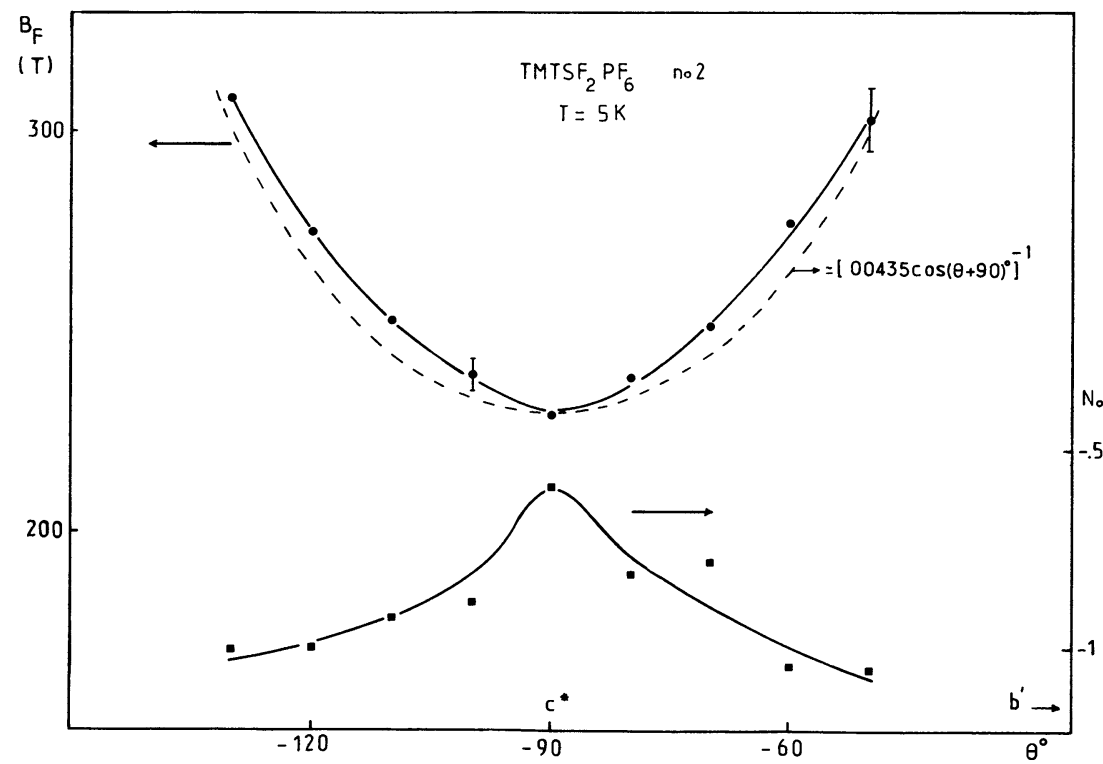

Fig. 6. - Angular dependence of the fundamental field $B_{\mathrm{F}}$ and of the phase $N_{0}$ for $T=5 \mathrm{~K}$, on sample $\mathrm{n}^{\circ} 2$. 
b) A cylindrical FS extending along the $c^{*}$ axis would imply a cosine-type law of the form (drawn in dashed line in Fig. 5 and Fig. 6) :

$$
\frac{1}{B_{\mathrm{F}}}(\theta)=\frac{1}{B_{\mathrm{F}}}\left(-90^{\circ}\right) \cos \left(\theta+90^{\circ}\right) \text {. }
$$

A significant deviation from this law is observed for the two samples with the same order of magnitude. The determined values of $B_{\mathrm{F}}$ are systematically above the theoretical line. This means that the cross-section $S$ is always larger than that associated with the regular tube. The simplest interpretation of this deviation involves the warping of the FS along the $c^{*}$ axis related to the transverse integral $t_{c}$. In this case the Fermi orbit corresponds to a minimal cross-section as shown in figure 7. A simple model can give an estimate of the transverse integral $t_{c}$ from the diffe-

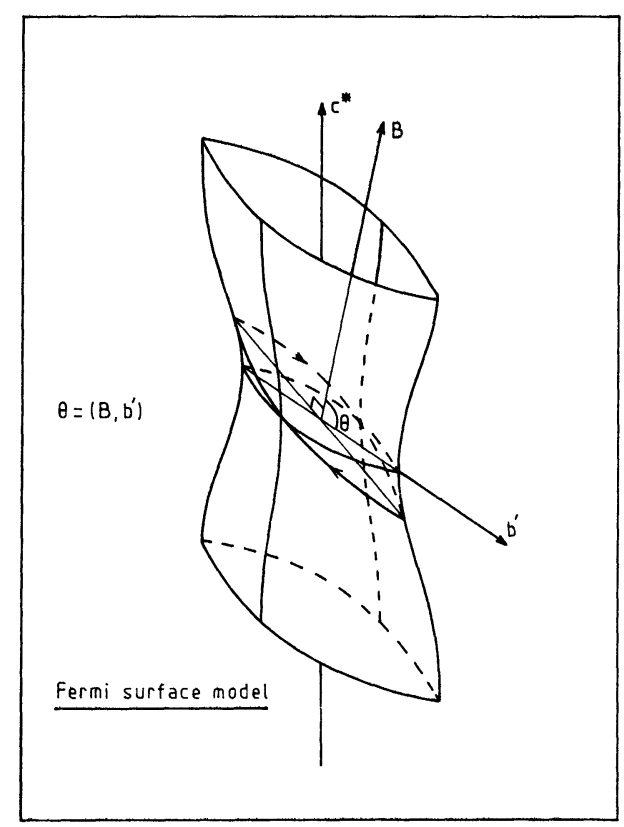

Fig. 7. - Warped Fermi surface model showing closed orbits in planes perpendicular to $B$.

rence $\Delta B_{\mathrm{F}}$ between the experimental and the theoretical $B_{\mathrm{F}}$ (cosine law) since, in the regime where quantum oscillations are observed, the Fermi energy of the « semi-metal " amounts to $\approx t_{b}[12]$, we can say very crudely that :

$$
\frac{t_{c}}{t_{b}} \approx \frac{\Delta B_{\mathrm{F}}}{B_{\mathrm{F}}}
$$

The maximum value for $\Delta B_{\mathrm{F}} / B_{\mathrm{F}}$ being around $3 \%$ and using the value of P. M. Grant for $E_{\mathrm{F}}$ at low temperature $\left(E_{\mathrm{F}} \simeq 279 \mathrm{meV}\right)$, with $t_{a} / t_{b} \simeq 10$ [13], we find $t_{c} \simeq 0.7 \mathrm{meV}$. This result is in a fair agreement with values obtained from conductivity anisotropy experiments [14].

c) The study of the angular variation of the phase $N_{0}$ of the oscillations (Fig. 5 and Fig. 6) gives a confirmation of the FS curvature [15].

Moreover no shift of the oscillation positions is observed when the temperature is varied. So the number of carriers is assumed to be constant in the region where the SdH effect is visible 
$(9 \mathrm{~K}-4.6 \mathrm{~K})$. The only parameters which can vary with temperature are the mobility $\mu$ and the effective mass $m^{*}$ in relation with the increase of the resistivity $\rho$ with decreasing temperature in the SDW phase. The study of the variation of the oscillation amplitudes, $A_{T}$, with temperature and quantum number allows us to calculate the effective mass $m^{*}$. Here, if we suppose that $m^{*}$ and $\mu$ can be modified by the temperature we have to compare these amplitudes at a fixed temperature. The oscillatory part of the magnetoresistance for a three-dimensional model is given by [16] :

$$
A_{T}=\left(\Delta \rho / \rho_{0}\right)_{T} \sim \frac{T}{B^{1 / 2}} \frac{u(T)}{\operatorname{sh} u(T)} \exp \left(-\frac{\pi}{\mu(T) . B}\right)
$$

where

$$
u(T)=\frac{2 \pi^{2} k T}{\hbar}=\frac{2 \pi^{2} k T m^{*}}{\hbar e B} .
$$

By the comparison of two amplitudes we obtain the equation of the form :

$$
\begin{gathered}
T=\text { Const. } \\
\frac{A_{1}}{A_{2}}\left(\frac{B_{1}}{B_{2}}\right)^{3 / 2} \operatorname{sh} x-\operatorname{sh}\left(x \frac{B_{1}}{B_{2}}\right) \exp \frac{y}{B_{1}}\left(\frac{B_{1}}{B_{2}}-1\right)=0
\end{gathered}
$$

with

and

$$
x=u_{1}(T) ; \quad u_{2}(T)=x \frac{B_{1}}{B_{2}}
$$

$$
y=\frac{\pi}{\mu(T)} .
$$

Chaikin et al. have performed Hall measurements which provide an evaluation of the mobility at low temperature. They give $\mu \simeq 10^{5} \mathrm{~cm}^{2} / \mathrm{V} . \mathrm{s}[17]$. This implies a value $y \simeq 0.3 \mathrm{~V} . \mathrm{s} / \mathrm{m}^{2}$. Using different $y$ between 0.1 and $1 \mathrm{~V} . \mathrm{s} / \mathrm{m}^{2}$, the effective mass $m^{*}$ verifies :

$$
2 \lesssim \frac{m^{*}}{m_{0}} \lesssim 3 \quad\left(m_{0}=\text { free electron mass }\right) .
$$

The cyclotron mass we find does not seem to be in disagreement with Jacobsen's determinations made by reflectance measurements which give $m^{*}(a) \simeq m_{0}$ and $m^{*}\left(b^{\prime}\right) \simeq 20 m_{0}$ [18].

The thermal behaviour of the SdH, quite normal in the range $9 \mathrm{~K}-5 \mathrm{~K}$ with an increase of oscillation amplitudes when $T$ decreases, becomes rather strange for a very small variation of temperature. Below $5 \mathrm{~K}$ we observe at the same time a sharp disappearance of the oscillations and a rapid decrease of the previously large magnetoresistance. At $4.2 \mathrm{~K}$, the magnetoresistance is perfectly saturated at high fields and oscillations are no longer seen. These two phenomena are in agreement with a possible phase transition towards an open FS, occurring between 5 and $4.2 \mathrm{~K}$.

\section{Acknowledgments.}

We are very grateful to Dr D. Jérome and Dr M. Ribault (Laboratoire de Physique des Solides, Orsay, France) for their precious help in the interpretation of our results and their constant support during this work. 


\section{References}

[1] Jérome, D., Mazaud, A., Ribault, M. and Bechgaard, K., J. Physique Lett. 41 (1980) L-95.

[2] BechgaArd, K., Jacobsen, C. S., Mortensen, K., Pedersen, H. J. and Thorup, N., Solid State Commun. 33 (1980) 1119.

[3] Walsh Jr, W. M., Wudl, F., Thomas, G. A., Nalewajek, D., Hauser, J. J., Lee, P. A. and Poehler, T., Phys. Rev. Lett. 45 (1980) 829.

[4] Kwak, J. F. and Shirber, J. E. and Greene, R. L. and Engler, E. M., Phys. Rev. Lett. 46 (1981) 1296.

[5] Heritier, M., Montambaux, G. and Lederer, P., J. Physique Lett. 45 (1984) L-943.

[6] Gor'Kov, L. P. and Lebed', A. G., J. Physique Lett. 45 (1984) L-433.

[7] Chaikin, P. M., Choi, M. Y., Kwak, J. F., Brooks, J. S., Martin, K. P., Naughton, M. J., Engler, E. M. and Greene, R. L., Phys. Rev. Lett. 51 (1983) 2333.

[8] Ulmet, J. P., Auban, P. and Askenazy, S., Solid State Commun. 52 (1984) 547.

[9] Askenazy, S., The application of high magnetic fields in semiconductor physics (Proc. M.F.S.P., Oxford) 1978, p. 101.

[10] KwaK, J. F., J. Physique Colloq. 44 (1983) C3-839.

[11] Kwak, J. F., Phys. Rev. B 28 (1983) 3277.

[12] Jérome, D. and Schulz, H. J., Adv. Phys. 31 (1982) 299.

[13] Grant, P. M., Phys. Rev. B 26 (1982) 6888.

[14] Jacobsen, C. S., Mortensen, K., Weger, M. and Bechgaard, K., Solid State Commun. 38 (1981) 423.

[15] Onsager, L., Philos. Mag. 43 (7) (1952) 1006.

[16] Roth, L. and Argyres, P: N., Semicond. and Semimetals, Vol. 1, Chap. 6, Willardson and Beer, eds. (Acad. Press) 1966.

[17] Chaikin, P. M., Haen, P., Engler, E. M. and Greene, R. L., Phys. Rev. B 24 (1981) 7155.

[18] Jacobsen, C. S., Tanner, D. B. and BechgaArd, K., Phys. Rev. Lett. 46 (1981) 1142. 